\title{
Identification of Institutional Characteristics that May Drive (Avoidable) Hospitalization of Nursing Home Residents
}

\author{
Zhe Hong \\ University of Rochester \\ zhe.hong@simon.rochester.edu
}

\begin{abstract}
Avoidable hospital transfers increase the risk of negative outcomes for patients and cause waste in medical resources. While reducing emergency department (ED) transfer and hospitalization rate has been widely discussed, few IS scholars pay close attention to the concept of avoidable transfers. The paper, rooted in the context of nursing homes, provides a comprehensive review of the concept, identification, and contributing factors. In addition, empirical evidence is presented to complement the study. To the best of our knowledge, this is the first empirical work using nationwide aggregate data to study the effect of institutional characteristics on avoidable hospitalization from nursing homes. We found that while caregiver/resident ratio remains a heated topic in public policy debate, only a high intensity of service provided by well-trained medical practitioners (registered nurses and physicians) is associated with low levels of avoidable ED visits and hospitalization.
\end{abstract}

\section{Introduction}

In 2014, there were 15,600 nursing homes nationwide, with 1.7 million licensed beds [1]. Nursing homes serve those who need a short stay or residents with chronic conditions that require long-term care [2]. Reimbursement comes chiefly from Medicaid and Medicare. In general, Medicare pays for short-term, post-acute care and rehabilitation after a hospital stay, and Medicaid, which covers $60 \%$ of nursing home residents at an outlay of nearly $\$ 60$ billion dollars each year [3], pays for long-term care [4].

In the long-term care sector, residents may prefer nursing homes over home or residential health care because they need regular attention from health practitioners due to severe health problems, functional deficits, or disability [5]. Indeed, $50.4 \%$ of this population have been diagnosed with Alzheimers disease, $48.7 \%$ have depression, and $32.4 \%$ live with

\author{
Abraham Seidmann \\ University of Rochester \\ avi.seidmann@simon.rochester.edu
}

diabetes [1]. In addition, about $85 \%$ are over 65 and about $40 \%$ are over 85 [1]. A majority of the residents also need assistance in aspects of daily living such as bathing, dressing, walking, and eating.

In the short-term care sector, patients discharged from acute care in a hospital may be transferred to nursing homes for transitional care. The average stay per Medicare admission is 25.9 days, and $83.6 \%$ of those admitted are discharged before day 40 [6]. The short stay can adversely affect care quality: as patients transit between different organizations, they may experience delays in follow-up, inadequate exchang of information, and conflicting or inappropriate health advice.

Although nursing homes feature 24-hour services, with an average of 3.88 nursing hours per resident per day [1], adverse events happen rather frequently. When the situation is deemed urgent, a resident will be transferred to a hospital immediately. The most common reasons for transfer include abdominal pain, abnormal vital signs, altered mental state, shortness of breath, and uncontrolled pain [7]. The annual hospitalization rate per bed (once a resident is transferred or discharged, a bed will be reassigned to an incoming resident) varies between 0.16 and 1.49 for different nursing homes [8] . According to a study by the US Office of Inspector General on Medicare-certified nursing homes [9], about one quarter of their residents were admitted to hospitals in fiscal year 2011 , of which $67.8 \%$ were transferred once, $20 \%$ were transferred twice, and $12.2 \%$ were transferred three or more times. One resident hospitalization episode is estimated to cost Medicare $\$ 11,255$, resulting in annual expenditures of $\$ 14.3$ billion. The study also listed common reasons for those hospitalizations. Septicemia accounted for $13.4 \%$ of visits and $20.8 \%$ in hospital reimbursements. Other reasons (ordered by percentage of hospitalization) were pneumonia, congestive heart failure, urinary tract infections, aspiration issues, acute renal failure, complication of device, and respiratory failure/insufficiency.

Unnecessary hospitalizations increase the risk of 
negative outcomes for patients, by disrupting care plans and inducing stress and iatrogenic illness [9]. Research indicates that a substantial portion of the transfers that occur are inappropriate or avoidable [10]. Thus, avoiding unneeded transfers can provide better health care to patients and at the same time reduce costs for insurers. Telemedicine may offer an effective way to achieve this goal. Since inappropriate hospitalization can be attributed to insufficient expertise of the on-site staff, access to physicians during off hours and other telemonitoring practices may improve care quality [11]. Despite the efforts made by IS scholars to study the effect of health IT on care quality, most studies are centered on the general transfer rate, with little attention to avoidable hospitalization rate, a concept widely discussed and adopted in medical literature.

This paper reviews extant literature on the conceptual framework of avoidable hospitalization, with an emphasis on its identification, and then moves to summarizing factors affecting the transfer decision. In addition, empirical evidence is presented to complement the study. We found that while caregiver/resident ratio remains a heated topic in public policy debate, only a high intensity of service provided by well-trained medical practitioners (registered nurses and physicians) is associated with low levels of avoidable ED visits and hospitalization.

The contributions of this paper are twofold: first, using both theory and empirical evidence, it provides a comprehensive review of contributing factors on avoidable hospital transfers, shedding light on the path to better and cost-effective care; second, to the best of our knowledge, this is the first empirical work using nationwide aggregate data to study the effect of institutional characteristics on avoidable hospitalization from nursing homes.

\section{Research Method}

The review seeks to cover articles published between 1990 and 2019. The following search procedure is followed to select articles in this field:

- Initial search was conducted in 5 major electronic health databases: CINAHL, MEDLINE, EMBASE, SCOPUS, and PROQUEST.

- The search was based on keywords avoidable/unneeded/unnecessary transfers or hospitalizations. To confine the research scope to nursing homes, we also use the keywords nursing home, long term care facility, short term care facility, extended care facility, continuing care facility.
- We stretched the search to bibliographies and references, covering relevant literature that might not include the keywords directly.

- We carefully reviewed the abstract and citations of each article.

- The papers were then selected based on their credibility, influence, relevance, and timeliness.

- To extract contributing factors, we used content analysis to synthesize the data. Factors with similar dimensions and definitions were merged into one.

Thirty-two journal articles were found after the search-and-filter process. Most of the papers are from Journal of the American Medical Directors Association (JAMDA), Health Affairs, The New England Journal of Medicine, and Journal of the American Geriatrics Society (JAGS).

\section{Conceptual Framework}

Although there lacks a rigorous and universal definition on avoidable transfer, we found that hospitalizations or transfers with the following characteristics are in most cases deemed as avoidable:

- The condition of the resident is of low-acuity [12]: most of the transferred patients in this category were quickly discharged after receiving elementary test and treatment to stabilize the condition.

- The hospitalization can be prevented with adequate preventive care $[13,14]$.

- The hospitalization is due to a failure of management in early-acute or low-acuity symptoms [15].

- The hospitalization, in expectation, cannot improve the clinical outcomes [16].

- Inducing worse end of life outcomes when maximizing comfort has been expressed as the primary goal [17].

- Most readmissions within 30 days are considered avoidable [18].

The various definitions of avoidability underscore the importance of analyzing and identifying avoidable hospital transfers in a systematic way. In the next section, we will elucidate how scholars identify the avoidability and the potential pitfalls of each approach. 
Table 1. Medical Conditions Associated with Avoidable Hospitalization

\begin{tabular}{ll}
\hline Medical Condition & ICD-9-CM \\
\hline Immunization-preventable conditions & $033,037,390,391,320.0$ \\
Congenital syphilis & 090 \\
Grand mal seizure disorders & $345,780.3$ \\
Severe ear, nose, and throat infections & $382,462,463,465,472.1$ \\
Tuberculosis & $011-018$ \\
Chronic obstructive pulmonary disease $_{\text {Bacterial pneumonia }}{ }^{2}$ & $466,491,492,494,496$ \\
Asthma $_{\text {Congestive heart failure }}{ }^{\text {a }}$ & $468,481,482.2,482.3,482.9,483$ \\
Hypertension & 493 \\
Angina & $428,518.4$ \\
Cellulitis & $401.0,401.9,402.0,402.1,402.9$ \\
Diabetes wit ketoacidosis or hypersmolar coma & $411.1,411.8,413$ \\
Diabetes with specified manifestations & $681,682,683,686$ \\
Diabetes without specified complications & $250.1-250.3$ \\
Hypoglycemia & $250.8,250.9$ \\
Gastroenteritis & 250.0 \\
Kindey/urinary tract infection & 251.2 \\
Dehydration & 588.8 \\
Iron deficiency anemia & $590,599.0,599.9$ \\
Nutritional deficiency & 276.5 \\
Failure to thrive & $280.1,280.8,280.9$ \\
Pelvic inflammatory disease & $260-262,268.0,268.1$ \\
Dental conditions & 783.4 \\
\hline
\end{tabular}

\section{Methodology on Identification}

The estimated avoidable hospital transfer rates vary dramatically in literature: ranging from $20 \%$ to $80 \%$. While a portion of such variation can be explained by nursing home and patients heterogeneity, a significant portion should be attributed to the identification methods deployed. This section reviews the general approaches on identifying the avoidability. The official guideline on identifying avoidable hospitalization is ambulatory care-sensitive hospitalizations (ACSH), which listed conditions recognized as preventable with timely treatment and resources. Common conditions include grand malseizure disorders, ear, nose and throat infections, tuberculosis, chronic obstructive pulmonary disease, bacterial pneumonia, asthma, congestive heart failure, hypertension, and certain type of diabetes. Table 1 provides a more detailed description with ICD-9 code [19].

CMS also listed six conditions, which are pneumonia, dehydration, congestive heart failure, urinary tract infection, skin ulcers, COPD/asthma, asserting that they contributed to $80 \%$ of the avoidable hospitalization in long term care facilities. Although listed conditions can provide an estimate for the avoidable transfers, those guidelines are insufficient to assess the avoidability on the individual level. We also introduce how avoidability is measured in most medical studies.

The primary approach is called retrospective review. Two branches of reviewing methods are commonly seen in the literature. The first branch compares the ED visits by those that did not lead to hospital admission with those that lead to admissions. The data is acquired either from nursing homes participating in the research or the National Hospital Ambulatory Care Survey. Despite the large number of samples, such methodology only covers the situation of avoidable hospital transfer of non-emergent case. A significant portion of preventable cases, which arise as a failure of proper chronic disease management and timely treatment in nursing home, remain undiscovered. An alternative approach is through comprehensive retrospective review of patients medical history and communication history. The study randomly selects a number of patients who have been transferred to ED in the past months. Then a group of experts, usually composed of one or several trained physicians, one experienced nurse from care facility, and one expert specializing in geriatrics care, are recruited. The experts will scrutinize the available medical records, with special notice to baseline health status, characteristics of acute symptoms (including lab results and vital signs), known interventions, response to on site treatment, and resource required. In some studies, reviewers also interview patients and their families on the attitude of hospital transfer. The appropriateness of a transfer is denied when the reviewers unanimously agreed on its avoidability. For better credibility, the conclusion is validated by outside medical practitioners who are not involved in the study. In certain cases, sensitivity analysis is also carried out, requiring the results to be robust under slight variation of data.

Despite the comprehensiveness of the above identification strategy, such approach still suffers major limitations [20]. First, the evaluation is still subject to personal judgment or expertise. Doctors in different regions or with different backgrounds can give distinct conclusions. Second, the process is time consuming and costly, requiring high level of human input, hence quite unlikely to scale. Third, patient samples are drawn either from a city or from several cities in a state, therefore the representativeness is under doubt. Realizing those constraints of this approach, in this paper we use nationwide aggregate data on nursing homes to investigate the effect of institutional characteristics on avoidable transfers and hospitalization. Our approach, differing from the current path in literature, covers a wider range of nursing facilities and limits the sampling error. The details will be presented in section 6 .

\section{Contributing Factors}

Before detailing the multi-dimensional contributing factors of the avoidable hospital transfer, we will briefly introduce how those factors are discovered.

The prevalent approach is through root cause analysis. After a group of experts have reached a unanimous conclusion on the avoidability of transfer, both qualitative and quantitative approaches are 
deployed to identify the root causes. For example, [16] compared the characteristics between transfers rated as avoidable with those rated as appropriate and presented the characteristics that are significantly different. Other studies use statistical tools such as Cox proportional hazard regression [21].

In absence of the expert opinion on appropriateness, directly surveying the medical practitioners on causes of preventable hospital transfers are also feasible. In this paper, we summarize the contributing factors for avoidable transfers observed in the past literature and brief potentially valid explanations.

- Lack of Communication: [22] indicated that both doctors and nurses ranked the patient preference as the most important consideration in the decision-making process. In fact, nursing homes are consistently understaffed, so the avoidable transfer may be attributed to a lack of prior communication with patients on their conditions. Most patients are inclined to think transferring to ED is best for their health in all circumstances.

- Low care quality: many transfers can be prevented if the nursing home offers a high level of care, which requires sufficient discharge communication with the hospital, planned care with scheduled follow ups, frequent primary care visits, and routine readmission risk assessment [23]. Some research also shows that allowing nursing home stuff to communicate with the hospital staff face-to-face is more efficient than letting them read the electronic medical records. Effective Communication can ensure consistent and planned care.

- Untrained on-site stuff: Many transfers are made by nurses instead of primary physicians. Nurses may lack the expertise or confidence to stabilize the patient's condition, therefore having to transfer residents to emergency room [24]. In fact, the majority of residents are referred to ED outside normal working hours, when physicians are less likely to be on site [25]. Nevertheless, both increasing the staff level of physicians and nurses can reduce avoidable hospital transfer as they both provide high quality regular care [22].

- Lack of Transition Model: : [22, 26] underscore that despite the availability of evidence-based guidelines such as the INTERACT model, some nursing homes are reluctant to embrace those established instructions. This phenomenon may be attributed to unfamiliarity to tools, lack of time for training, and fear to be held accountable as those models entail a track of records on decision making.

- Lack of on-site facility: Many nursing home residents are sent to ER and quickly discharged after running simple tests. In this population, many are transferred to ER after a fall and are discharged after CT scan [27, 28]. It appears that since nursing homes do not have the needed medical equipment, they are transferring patients for tests instead of emergency treatment. In addition, since antibiotics require doctor's prescription, some nursing homes have a rather low level of stock and diversity on such medicine, delaying the time that patients can get the proper medication in case of infection. This finding is consistent with the finding in [29], which asserts that the transfer is more likely to be inappropriate when little or no intervention is tried in the care facility.

- Patient's Cognitive Ability: Some studies [30] also emphasize certain populations are more likely to suffer unnecessary hospitalization. [16] found that patients with advanced dementia, whose cognitive and functional abilities are significantly compromised by the disease, have an estimated avoidable hospitalization rate of 75 percent.

- Patient's baseline condition: A baseline condition refers to the patient's health status when transitioned to the care facility. Although a transfer decision should be made based on how the patient's condition can be improved after hospitalization, [29] shows that residents with poor baseline health are more likely to incur inappropriate transfer. This, with previous evidence presented in the article, highlights the need for a systematic evaluation before transferring nursing home residents to ED.

- Insurance Status: [31] observed that nursing home residents with Medicaid are more likely to be transferred compared with those with private insurance. The gap might be driven by financial incentives since the insurance paying system makes it less costly to transfer Medicaid residents to ED rather than treat them on site.

- Others: government legislation, bureaucratic concerns ... [32] 


\section{Empirical Evidence}

Beyond reviewing the past literature, we seek using real world data to validate or contradict some of the previous findings. As an ongoing research, this paper will focus the lens at the effect of institutional characteristics on hospitalization. The data is obtained from files in nursing home compare initiated by CMS. Since CMS revised its data file format a few times, we use the data published in April 2019, which measures the period from 2017.01.01 to 2018.09.30, for summary statistics and cross sectional analysis.

Table 3 in Appendix presents the summary statistics for care facilities. In the short term care sector, the average rehospitalization rate is $22.8 \%$, with a slight drop to $22.6 \%$ after adjusting for risk factors; the average ED transfer rate is $11.1 \%$, with a slight drop to $10.7 \%$ after adjusting for risk factors. The stark reality is that the maximum rates for rehospitalization and ED visits are exceeding or near 50\%, raising serious concerns on the service quality in some care facilities. In the long term care sector, per 1000 resident days, there are on average 1.8 hospitalization incidents and $1.5 \mathrm{ED}$ visits. The numbers drop to 1.7 and 1.0 correspondingly after adjusting for risks.

The number of care facilities also varies dramatically in different states. Topping the list is Texas, with 1225 registered care facilities, followed by California with 1197 nursing homes. Ohio, Illinoi, Pennsylvania, Florida, New York, Indiana, and Missouri, have over 500 nursing homes.

The challenge in analyzing the contributing factors of avoidable transfer is disentangling the effect driven by the patient's health status. To study the influence of nursing home institutional characteristics on the avoidable ED visits and hospitalization, we use the risk adjusted rate as our dependent variable. Since those rates already account for the patient characteristics, they should be reliable proxies for our goal. Moreover, since those are percentage based, the size of nursing facilities only influences those variables through care quality.

We include the provider information dataset from the nursing home compare program. The date duration is the same as previous dataset to ensure consistency. See table 4 in appendix for summary statistics.

There are 15563 providers in total. On average, each nursing facility devotes 3.87 total staffing hours per resident per day, of which only $1 / 6$ comes from registered nurses. The physician hour for each resident per day is 0.082 . Approximately half of the caring facilities have received fines and on average 1.2 facility incidents have been reported during the period. The variable name illustration can be found in table 2 .
Table 2. Medical Conditions Associated with Avoidable Hospitalization

\begin{tabular}{|c|c|c|}
\hline $\begin{array}{l}\text { Variable Name (column headers on } \\
\text { CSV Downloadable files) }\end{array}$ & Description & Format/Values \\
\hline PROVNUM & Federal Provider Number & 6 alphanumeric characters \\
\hline STATE & Provider State & 2-character postal abbreviation \\
\hline BEDCERT & Number of Federally Certified Beds & jinteger \\
\hline RESTOT & $\begin{array}{l}\begin{array}{l}\text { Average number of residents based on MDS } \\
\text { daily census }\end{array} \\
\end{array}$ & number with one decimal place 000. \\
\hline AIDHRD & $\begin{array}{l}\text { Reported Nurse Aide Staffing - Hours per } \\
\text { Resident per Day }\end{array}$ & real number, up to 5 decimal places \\
\hline VOCHRD & $\begin{array}{l}\text { Reported LPN Staffing - Hours per Resident } \\
\text { per Day }\end{array}$ & real number, up to 5 decimal places \\
\hline RNHRD & $\begin{array}{l}\text { Reported RN Staffing - Hours per Resident } \\
\text { per Day }\end{array}$ & real number, up to 5 decimal places \\
\hline TOTLICHRD & $\begin{array}{l}\text { Reported Licensed Staffing - Hours per } \\
\text { Resident per Day (RN + LPN) }\end{array}$ & real number, up to 5 decimal places \\
\hline TOTHRD & $\begin{array}{l}\text { Reported Total Nurse Staffing - Hours per } \\
\text { Resident per Day (Aide+LPN+RN) }\end{array}$ & real number, up to 5 decimal places \\
\hline PTHRD & $\begin{array}{l}\text { Reported Physical Therapy Staffing - Hours } \\
\text { per Resident Per Day }\end{array}$ & real number, up to 5 decimal places \\
\hline cycle_1_defs & $\begin{array}{l}\text { Total Number of Health Deficiencies in } \\
\text { Rating cycle } 1\end{array}$ & integer \\
\hline cycle_2_defs & $\begin{array}{l}\text { Total Number of Health Deficiencies in } \\
\text { Rating cycle 2 - See CMS 5-Star Techinical }\end{array}$ & linteger \\
\hline cycle_3_defs & \begin{tabular}{|l|} 
Total Iumber of Health Deficiencies in \\
Rating cycle 3 - See CMS 5-Star Techinical \\
Users' Guide for description of Rating cycles
\end{tabular} & integer \\
\hline incident_cnt & Number of Facility-Reported Incidents & integer \\
\hline cmplnt_cnt & Number of Substantiated Complaints & linteger \\
\hline FINE_CNT & Number of Fines & integer \\
\hline FINE_TOT & Total Amount of Fines in Dollars & integer \\
\hline PAYDEN CNT & Number of Payment Denials & integer \\
\hline
\end{tabular}

We then run linear regression to see how institutional factors contribute to the avoidable hospitalization. Since all four dependent variables are proxies for the avoidable transfers or hospitalizations, we are interested in the institutional factors that are significant in all four regressions. See results in table 5 in appendix.

The first observation is that there does not exhibit state level differences on the ED visits but there does exist gaps in hospitalization rates. This might be explained by different standards in hospitalization among different states.

Second, nursing facilities with fewer beds tend to have better care quality, reducing the rate of ED visits and hospitalization in both long term and short term care sector. The economies of scale principle does not seem to apply in this context, as when controlling the care intensity (measured by hours spent per resident per day), smaller nursing homes tend to achieve higher efficiency in care quality. Moreover, our finding is consistent with the prior literature conjecturing the lack of trained staff contributes to the avoidable transfers. Although in theory residents have better care with more attention from licensed staff, the evidence suggests that only the hours spent with registered nurses and physicians can significantly reduce the transfer and hospitalization rate in all context. In the short term care sector, one more hour per resident by registered nurses will lower the ED visit rate by 1.5 percent and the rehospitalization rate by 1.1 percent. In the long term care sector, one more hour per resident by registered nurses will lower the ED visit rate by 0.4 per 1000 resident days and the hospitalization rate by 0.5 per 1000 resident days. The value of physician input is even more pronounced. In the short term care sector, one more hour per resident by 
physicians will lower the ED visit rate by 4.2 percent and the rehospitalization rate by 3.2 percent. In the long term care sector, one more hour per resident by physicians will lower the ED visit rate by 1.2 per 1000 resident days and the hospitalization rate by 0.35 per 1000 resident days. This observation calls for more trained staff, with sufficient medical knowledge, to plan and manage residents' health on a regular basis.

It is noteworthy that the operational effectiveness can be measured by how many incidents (mainly injuries inside nursing home) are reported, how much fines are received, and how many health deficiency notices are issued. In our study, none of those factors have significant effects across all situations considered. The only partial exception is the total fine counts: caring facilities subject to more frequent fines have higher level of ED transfers, but such effect disappears for the hospitalization measure.

We also conducted robustness check by introducing non-linearity, using clustered errors on state level, the finding proves to be robust under different model specifications.

\section{Conclusion}

Our paper is the first IS paper systematically looking at the definition, identification, and contributing factors of avoidable transfers and hospitalization. We reviewed extensively on the medical literature in this context, summarizing the past findings and admitting their limitations. The previous research, constrained by the scope of data available, uses data collected from a few participating nursing homes and requires expert judgment on the appropriateness of the decision made. Our empirical research utilizes nationwide aggregate level data to study the effect of institutional characteristics on avoidable hospitalization from nursing homes. Since the data duration lasts more than a year and the dependent variable is averaged, our data suffers little noise from sampling error or subjective variation, and is thus more accurate and reliable. We found that while caregiver/resident ratio remains a heated topic in public policy debate, only a high intensity of service provided by well-trained medical practitioners (registered nurses and physicians) is associated with low levels of avoidable ED visits and hospitalization. In the current stage, the binding constraint to a more effective and higher quality nursing home care is still the lack of registered nurses and physicians. Nursing homes should either initiate stuff training program to increase the knowledge and experience of all licensed care givers, or introduce tools like health IT, which allow remote monitoring, diagnosis and treatment, to boost productivity of the registered nurses and physicians.

\section{Limitation}

This is an ongoing research and there is much more we would like to explore in the future. The major limitation is that the aggregate level data obstructs us from observing the effect of socio-demographic factors. Nevertheless, we are confident that a better understanding on the role of institutional characteristic can still shed light on future improvement of care quality and effectiveness.In the future, we seek to incorporate data spanning longer time horizon to complete the research. In addition, we plan to conduct the research at a more granular level, giving adequate consideration to the effect of zip code and the nursing home's neighboring environment.

\section{References}

[1] L. Harris-Kojetin, M. Sengupta, E. Park-Lee, R. Valverde, C. Caffrey, V. Rome, and J. Lendon, "Long-term care providers and services users in the united states: data from the national study of long-term care providers, 2013-2014.," Vital \& health statistics. Series 3, Analytical and epidemiological studies, no. 38, pp. x-xii, 2016.

[2] F. H. Decker, "Nursing homes, 1977-99; what has changed, what has not?: facts from the national nursing home surveys," 2005.

[3] KFF, "Medicaid's role in nursing home care," May 2018.

[4] P. Span, "In the nursing home, empty beds and quiet halls," The New York Times, 2018.

[5] M. Mjørud, M. Kirkevold, J. Røsvik, G. Selbæk, and $\mathrm{K}$. Engedal, "Variables associated to quality of life among nursing home patients with dementia," Aging \& mental health, vol. 18, no. 8, pp. 1013-1021, 2014.

[6] C. C. Quinn, C. L. Port, S. Zimmerman, A. L. Gruber-Baldini, J. D. Kasper, I. Fleshner, B. Yody, J. Loome, and J. Magaziner, "Short-stay nursing home rehabilitation patients: Transitional care problems pose research challenges," Journal of the American Geriatrics Society, vol. 56, no. 10, pp. 1940-1945, 2008.

[7] J. G. Ouslander, I. Naharci, G. Engstrom, J. Shutes, D. G. Wolf, M. Rojido, R. Tappen, and D. Newman, "Hospital transfers of skilled nursing facility (snf) patients within 48 hours and 30 days after snf admission," Journal of the American Medical Directors Association, vol. 17, no. 9, pp. 839-845, 2016.

[8] B. Graverholt, T. Riise, G. Jamtvedt, B. S. Husebo, and M. W. Nortvedt, "Acute hospital admissions from nursing homes: predictors of unwarranted variation?," Scandinavian journal of public health, vol. 41, no. 4, pp. 359-365, 2013.

[9] D. R. Levinson and I. General, "Medicare nursing home resident hospitalization rates merit additional monitoring," US Office of Inspector General, 2013.

[10] J. G. Ouslander and R. A. Berenson, "Reducing unnecessary hospitalizations of nursing home residents," 
New England Journal of Medicine, vol. 365, no. 13, pp. 1165-1167, 2011.

[11] D. C. Grabowski and A. J. OMalley, "Use of telemedicine can reduce hospitalizations of nursing home residents and generate savings for medicare," Health Affairs, vol. 33, no. 2, pp. 244-250, 2014.

[12] O. Intrator, J. Zinn, and V. Mor, "Nursing home characteristics and potentially preventable hospitalizations of long-stay residents," Journal of the American Geriatrics Society, vol. 52, no. 10, pp. 1730-1736, 2004.

[13] A. Gadomski, P. Jenkins, and M. Nichols, "Impact of a medicaid primary care provider and preventive care on pediatric hospitalization," Pediatrics, vol. 101, no. 3, p. e1, 1998.

[14] G. Arendts, T. Reibel, J. Codde, and J. Frankel, "Can transfers from residential aged care facilities to the emergency department be avoided through improved primary care services? data from qualitative interviews," Australasian Journal on Ageing, vol. 29, no. 2, pp. 61-65, 2010.

[15] Y. Eggli, B. Desquins, E. Seker, and P. Halfon, "Comparing potentially avoidable hospitalization rates related to ambulatory care sensitive conditions in switzerland: the need to refine the definition of health conditions and to adjust for population health status," BMC health services research, vol. 14, no. 1, p. 25, 2014

[16] M. W. Carter and F. W. Porell, "Vulnerable populations at risk of potentially avoidable hospitalizations: the case of nursing home residents with alzheimer's disease," American Journal of Alzheimer's Disease \& Other Dementias ${ }^{\circledR}$, vol. 20, no. 6, pp. 349-358, 2005.

[17] J. L. Givens, K. Selby, K. S. Goldfeld, and S. L. Mitchell, "Hospital transfers of nursing home residents with advanced dementia," Journal of the American Geriatrics Society, vol. 60, no. 5, pp. 905-909, 2012.

[18] J. Donzé, D. Aujesky, D. Williams, and J. L. Schnipper, "Potentially avoidable 30-day hospital readmissions in medical patients: derivation and validation of a prediction model," JAMA internal medicine, vol. 173, no. 8, pp. 632-638, 2013.

[19] M. W. Carter, "Factors associated with ambulatory caresensitive hospitalizations among nursing home residents," Journal of Aging and Health, vol. 15, no. 2, pp. 295-331, 2003.

[20] J. G. Ouslander and K. Maslow, "Geriatrics and the triple aim: Defining preventable hospitalizations in the long-term care population," Journal of the American Geriatrics Society, vol. 60, no. 12, pp. 2313-2318, 2012.

[21] M. Becker, T. Boaz, R. Andel, and A. DeMuth, "Predictors of avoidable hospitalizations among assisted living residents," Journal of the American Medical Directors Association, vol. 13, no. 4, pp. 355-359, 2012.

[22] D. C. Grabowski, K. A. Stewart, S. M. Broderick, and L. A. Coots, "Predictors of nursing home hospitalization: a review of the literature," Medical Care Research and Review, vol. 65, no. 1, pp. 3-39, 2008.

[23] G. H. Davidson, E. Austin, L. Thornblade, L. Simpson, T. D. Ong, H. Pan, and D. R. Flum, "Improving transitions of care across the spectrum of healthcare delivery: A multidisciplinary approach to understanding variability in outcomes across hospitals and skilled nursing facilities," The American Journal of Surgery, vol. 213, no. 5, pp. 910-914, 2017.
[24] G. Arendts, S. Quine, and K. Howard, "Decision to transfer to an emergency department from residential aged care: a systematic review of qualitative research," Geriatrics \& Gerontology International, vol. 13, no. 4, pp. 825-833, 2013.

[25] R. Briggs, T. Coughlan, R. Collins, D. O'Neill, and S. Kennelly, "Nursing home residents attending the emergency department: clinical characteristics and outcomes," QJM: An International Journal of Medicine, vol. 106, no. 9, pp. 803-808, 2013.

[26] J. G. Ouslander, M. Perloe, J. H. Givens, L. Kluge, T. Rutland, and G. Lamb, "Reducing potentially avoidable hospitalizations of nursing home residents: results of a pilot quality improvement project," Journal of the American Medical Directors Association, vol. 10, no. 9, pp. 644-652, 2009.

[27] R. E. Burke, S. P. Rooks, C. Levy, R. Schwartz, and A. A. Ginde, "Identifying potentially preventable emergency department visits by nursing home residents in the united states," Journal of the American Medical Directors Association, vol. 16, no. 5, pp. 395-399, 2015.

[28] M. Kirsebom, M. Hedström, B. Wadensten, and U. Pöder, "The frequency of and reasons for acute hospital transfers of older nursing home residents," Archives of gerontology and geriatrics, vol. 58, no. 1, pp. 115-120, 2014.

[29] D. Saliba, R. Kington, J. Buchanan, R. Bell, M. Wang, M. Lee, M. Herbst, D. Lee, D. Sur, and L. Rubenstein, "Appropriateness of the decision to transfer nursing facility residents to the hospital," Journal of the American Geriatrics Society, vol. 48, no. 2, pp. 154-163, 2000.

[30] K. S. Goldfeld, M. B. Hamel, and S. L. Mitchell, "The cost-effectiveness of the decision to hospitalize nursing home residents with advanced dementia," Journal of pain and symptom management, vol. 46, no. 5, pp. 640-651, 2013.

[31] S. Cai, S. C. Miller, D. L. Nelson, and D. B. Mukamel, "The impact of medicaid payer status on hospitalizations in nursing homes," Medical care, vol. 53, no. 7, p. 574, 2015.

[32] L. M. Trahan, J. A. Spiers, and G. G. Cummings, "Decisions to transfer nursing home residents to emergency departments: a scoping review of contributing factors and staff perspectives," Journal of the American Medical Directors Association, vol. 17, no. 11, pp. 994-1005, 2016. 


\section{Appendix}

Table 3. Summary Statistics ED and Hospitalization

\begin{tabular}{lccccccc}
\hline \hline Statistic & $\mathrm{N}$ & Mean & St. Dev. & Min & Pctl(25) & Pctl(75) & Max \\
\hline Percent Rehospitalized Adjusted & 13,499 & 22.628 & 6.259 & 0.000 & 18.676 & 26.434 & 57.887 \\
Percent Rehospitalized & 13,499 & 22.821 & 7.316 & 0.000 & 18.056 & 27.273 & 68.000 \\
Percent ED Adjusted & 13,499 & 10.700 & 5.265 & 0.000 & 7.069 & 13.611 & 42.429 \\
Percent ED & 13,499 & 11.059 & 5.713 & 0.000 & 7.080 & 14.155 & 46.552 \\
LS hospitalized Adjusted & 13,768 & 1.749 & 0.769 & 0.000 & 1.218 & 2.183 & 10.727 \\
LS hospitalized & 13,768 & 1.824 & 0.983 & 0.000 & 1.148 & 2.309 & 12.106 \\
LS ED Adjusted & 13,768 & 1.028 & 0.677 & 0.000 & 0.560 & 1.328 & 6.421 \\
LS ED & 13,768 & 1.517 & 1.034 & 0.000 & 0.807 & 1.960 & 10.336 \\
\hline
\end{tabular}

Table 4. Summary Statistics Provider Info

\begin{tabular}{lccccccc}
\hline \hline Statistic & $\mathrm{N}$ & Mean & St. Dev. & Min & Pctl(25) & Pctl(75) & Max \\
\hline RESTOT & 15,502 & 85.588 & 52.928 & 1.000 & 50.625 & 107.600 & 751.800 \\
AIDHRD & 14,847 & 2.315 & 0.554 & 0.000 & 1.950 & 2.595 & 10.546 \\
VOCHRD & 14,847 & 0.877 & 0.368 & 0.000 & 0.658 & 1.066 & 4.852 \\
RNHRD & 14,847 & 0.677 & 0.522 & 0.000 & 0.387 & 0.806 & 7.504 \\
TOTLICHRD & 14,847 & 1.554 & 0.598 & 0.000 & 1.251 & 1.689 & 9.738 \\
TOTHRD & 14,847 & 3.869 & 0.939 & 1.504 & 3.314 & 4.216 & 15.069 \\
PTHRD & 14,847 & 0.082 & 0.102 & 0.000 & 0.025 & 0.101 & 2.493 \\
INCIDENT_CNT & 15,563 & 1.286 & 3.174 & 0 & 0 & 1 & 83 \\
CMPLNT_CNT & 15,563 & 4.353 & 7.604 & 0 & 0 & 5 & 95 \\
FINE_CNT & 15,563 & 0.470 & 0.814 & 0 & 0 & 1 & 7 \\
FINE_TOT & 15,563 & $16,065.390$ & $57,215.540$ & 0 & 0 & 7,803 & $1,508,727$ \\
PAYDEN_CNT & 15,563 & 0.103 & 0.359 & 0 & 0 & 0 & 4 \\
TOT_PENLTY_CNT & 15,563 & 0.574 & 1.000 & 0 & 0 & 1 & 9 \\
\hline
\end{tabular}


Table 5. Regression Results

\begin{tabular}{|c|c|c|c|c|}
\hline & $\begin{array}{c}(1) \\
\text { EDadjusted }\end{array}$ & $\begin{array}{c}(2) \\
\text { Hosadjusted }\end{array}$ & $\begin{array}{c}\text { (3) } \\
\text { LSEDadjusted }\end{array}$ & $\begin{array}{c}(4) \\
\text { LSHosadjusted }\end{array}$ \\
\hline State & $\begin{array}{c}0.00645 \\
(0.00344)\end{array}$ & $\begin{array}{c}-0.0323^{* * *} \\
(0.00412)\end{array}$ & $\begin{array}{c}0.000216 \\
(0.000418)\end{array}$ & $\begin{array}{c}-0.00749^{* * *} \\
(0.000484)\end{array}$ \\
\hline bedcert & $\begin{array}{l}0.0122^{* * *} \\
(0.00234)\end{array}$ & $\begin{array}{l}0.0125^{* * *} \\
(0.00280)\end{array}$ & $\begin{array}{l}0.00272^{* * *} \\
(0.000285)\end{array}$ & $\begin{array}{l}0.00235^{* * *} \\
(0.000329)\end{array}$ \\
\hline restot & $\begin{array}{c}-0.0304^{* * *} \\
(0.00259)\end{array}$ & $\begin{array}{c}-0.0155^{* * *} \\
(0.00310)\end{array}$ & $\begin{array}{c}-0.00582^{* * *} \\
(0.000315)\end{array}$ & $\begin{array}{c}-0.00301^{* * *} \\
(0.000364)\end{array}$ \\
\hline aidhrd & $\begin{array}{l}-0.111 \\
(0.108)\end{array}$ & $\begin{array}{c}-0.651^{* * *} \\
(0.129)\end{array}$ & $\begin{array}{r}-0.00873 \\
(0.0131)\end{array}$ & $\begin{array}{c}0.0173 \\
(0.0151)\end{array}$ \\
\hline vochrd & $\begin{array}{l}-0.415^{*} \\
(0.174)\end{array}$ & $\begin{array}{l}1.752^{* * *} \\
(0.208)\end{array}$ & $\begin{array}{c}-0.0597^{* *} \\
(0.0211)\end{array}$ & $\begin{array}{c}0.0862^{* * *} \\
(0.0244)\end{array}$ \\
\hline rnhrd & $\begin{array}{c}-1.545^{* * *} \\
(0.188)\end{array}$ & $\begin{array}{c}-1.087^{* * *} \\
(0.225)\end{array}$ & $\begin{array}{l}-0.400^{* * *} \\
(0.0228)\end{array}$ & $\begin{array}{c}-0.503^{* * *} \\
(0.0264)\end{array}$ \\
\hline pthrd & $\begin{array}{c}-4.233^{* * *} \\
(0.831)\end{array}$ & $\begin{array}{c}-3.162^{* *} \\
(0.994)\end{array}$ & $\begin{array}{c}-1.184^{* * *} \\
(0.101)\end{array}$ & $\begin{array}{c}-0.348^{* *} \\
(0.117)\end{array}$ \\
\hline cycle_1_defs & $\begin{array}{c}0.0184^{*} \\
(0.00852)\end{array}$ & $\begin{array}{l}0.00717 \\
(0.0102)\end{array}$ & $\begin{array}{l}0.000541 \\
(0.00103)\end{array}$ & $\begin{array}{l}0.00261^{*} \\
(0.00120)\end{array}$ \\
\hline cycle_2_defs & $\begin{array}{l}-0.00226 \\
(0.00946)\end{array}$ & $\begin{array}{l}-0.0172 \\
(0.0113)\end{array}$ & $\begin{array}{l}0.000284 \\
(0.00115)\end{array}$ & $\begin{array}{l}0.00418^{* *} \\
(0.00133)\end{array}$ \\
\hline cycle_3_defs & $\begin{array}{l}-0.00191 \\
(0.00889)\end{array}$ & $\begin{array}{l}-0.00679 \\
(0.0106)\end{array}$ & $\begin{array}{l}-0.000311 \\
(0.00108)\end{array}$ & $\begin{array}{c}0.00227 \\
(0.00125)\end{array}$ \\
\hline incident_cnt & $\begin{array}{c}0.0228 \\
(0.0163)\end{array}$ & $\begin{array}{c}-0.0898^{* * *} \\
(0.0195)\end{array}$ & $\begin{array}{l}-0.000535 \\
(0.00198)\end{array}$ & $\begin{array}{c}-0.0146^{* * *} \\
(0.00229)\end{array}$ \\
\hline cmplnt_cnt & $\begin{array}{l}-0.0166^{*} \\
(0.00829)\end{array}$ & $\begin{array}{l}0.0411^{* * *} \\
(0.00991)\end{array}$ & $\begin{array}{l}0.000356 \\
(0.00101)\end{array}$ & $\begin{array}{c}0.00204 \\
(0.00116)\end{array}$ \\
\hline fine_cnt & $\begin{array}{l}0.343^{* * *} \\
(0.0689)\end{array}$ & $\begin{array}{c}0.139 \\
(0.0824)\end{array}$ & $\begin{array}{l}0.0312^{* * *} \\
(0.00836)\end{array}$ & $\begin{array}{l}-0.00173 \\
(0.00967)\end{array}$ \\
\hline fine_tot & $\begin{array}{c}-3.81 \mathrm{e}-08 \\
(0.000000879)\end{array}$ & $\begin{array}{c}-0.000000214 \\
(0.00000105)\end{array}$ & $\begin{array}{c}0.000000324^{* *} \\
(0.000000107)\end{array}$ & $\begin{array}{c}7.27 \mathrm{e}-08 \\
(0.000000123)\end{array}$ \\
\hline payden_cnt & $\begin{array}{c}0.162 \\
(0.145)\end{array}$ & $\begin{array}{c}0.242 \\
(0.174)\end{array}$ & $\begin{array}{c}-0.000219 \\
(0.0176)\end{array}$ & $\begin{array}{c}0.0609^{* *} \\
(0.0204)\end{array}$ \\
\hline _cons & $\begin{array}{c}13.66^{* * *} \\
(0.330)\end{array}$ & $\begin{array}{c}23.73^{* * *} \\
(0.395)\end{array}$ & $\begin{array}{l}1.623^{* * *} \\
(0.0401)\end{array}$ & $\begin{array}{l}2.122^{* * *} \\
(0.0464)\end{array}$ \\
\hline $\begin{array}{l}N \\
\text { adj. } R^{2}\end{array}$ & $\begin{array}{l}11902 \\
0.051\end{array}$ & $\begin{array}{l}11902 \\
0.028\end{array}$ & $\begin{array}{l}11902 \\
0.125\end{array}$ & $\begin{array}{l}11902 \\
0.090\end{array}$ \\
\hline
\end{tabular}

